\title{
Impacto del Plan Nacional de Lectura y Bibliotecas (PNLB) sobre el número de libros leídos en Colombia*
}

\author{
Juan Felipe Parra Osorio \\ Economista de la Universidad del Valle, Santiago de Cali, Colombia \\ y Magister en Economía. Universidad de los Andes. Bogotá \\ Oscar Julián Corzo C. \\ Economista de la Universidad de Santander. Bucaramanga \\ y candidato a Magister en Economía. Universidad de los Andes. Bogotá
}

\begin{abstract}
El presente documento evalúa, a través de la metodología Propensity Score Matching, el impacto que ha tenido el Plan Nacional de Lectura y Bibliotecas - PNLBsobre la cantidad de libros que leen los colombianos. Las conclusiones arrojadas por el estudio permiten corroborar la veracidad de la hipótesis según la cual se afirma que, aquellos individuos pertenecientes a municipios beneficiados por el PNLB, tienen mejores hábitos de lectura que otros con características similares pero que no han sido objeto de atención. Así se proveen criterios objetivos que demuestran la conveniencia de fortalecer el programa en poblaciones ya beneficiadas, y de estimular a los munici-

* Los autores agradecen las sugerencias del calificador anónimo, así como los comentarios de Laura Cuesta, Nicolás Palau y Angélica Franco a una versión preliminar de este documento. Aplican los usuales descargos. El contenido de este documento es responsabilidad única de los autores.
\end{abstract}


pios que aún no se han beneficiado del Plan, para que cumplan con los requerimientos exigidos de manera que puedan ser elegibles.

\section{INTRODUCCIÓN}

El Plan Nacional de Lectura y Bibliotecas (PNLB) tiene su origen en el Plan Nacional de Desarrollo 2002-2006: «Hacia Un Estado Comunitario». Allí, el Gobierno colombiano propuso implementar programas nacionales o regionales que refuercen la identidad, las manifestaciones culturales y la creación de tejido social. En este contexto, se señala la lectura como una de las manifestaciones más contundentes de equidad en cuanto facilita el acceso a la información y al conocimiento. Además, se considera que tiene efectos sobre la convivencia, ya que promueve la comprensión entre las diferentes culturas; favorece el respeto a la diversidad, estimula la participación en la vida democrática y social del país y tiene incidencias positivas en la productividad de la población. En general, se considera que la lectura mejora la calidad de vida de las personas que encuentran en ella, un espacio de entretenimiento y la posibilidad de mejorar sus capacidades.

En virtud de estas características y ante la necesidad de un programa que incentive a la población para que mejore sus hábitos de lectura, surge el documento Conpes ${ }^{1} 3222$ de abril de 2003 que lleva por título: «Lineamientos del Plan Nacional de Lectura y Bibliotecas». A través de este documento, se le da respuesta a esta ordenanza y se promueve una política que busca, principalmente, hacer de Colombia un país de lectores.

En la búsqueda de estos objetivos generales se identificaron seis acciones prioritarias: (1) el fortalecimiento de las bibliotecas públicas, (2) la promoción y fomento de la lectura, (3) el mejoramiento de los circuitos de producción y circulación de libros, (4) la creación de un sistema de información, seguimiento y evaluación de la Red Nacional de Bibliotecas Públicas, (5) el establecimiento de un banco de proyectos y finalmente, (6) la elaboración de un programa de comunicación y medios.

El presente artículo se propone evaluar el primero de los componentes del PNLB que procura fortalecer las bibliotecas. Aunque éste no es el único objetivo del plan, si es el que mayores esfuerzos y recursos ha demandado, sin que hasta el momento se tenga

${ }^{1}$ El Consejo Nacional de Política Económica y Social-CONPES-, es la máxima autoridad nacional de planeación y se desempeña como organismo asesor del Gobierno colombiano en todos los aspectos relacionados con el desarrollo económico y social del país. 
una estimación de su impacto sobre la población beneficiaria en términos del número de libros leídos. Con este documento, es precisamente éste el vacío que se pretende llenar. Para esto, se realizará la estimación mediante la metodología Propensity Score Matching - PSM ${ }^{2}$, que posee unas características deseables en la medida en que soluciona algunos problemas típicos de la evaluación de políticas públicas.

Las dificultades más recurrentes cuando se intenta hacer un ejercicio de esta naturaleza, están relacionadas con la selección de la población beneficiaria. En esencia, cuando la selección de los beneficiarios no se realiza de manera aleatoria, se hace difícil la evaluación de programas de carácter social debido a que se requiere utilizar técnicas econométricas, que permitan determinar el efecto atribuible únicamente al plan. En el caso del PNLB, no se cumple la aleatoriedad requerida por cuanto la selección de los municipios beneficiados se hizo con base en el cumplimiento de una serie de requisitos ${ }^{3}$.

En consecuencia, el impacto del PNLB sobre el número de libros leídos por las personas que habitan los municipios que se han beneficiado, puede verse afectado por otras variables exógenas. Lo que se busca con esta evaluación de impacto es aislar esas otras variables, para calcular el efecto neto que ha tenido sobre los hábitos de lectura de los individuos, que resultan ser la unidad de análisis del presente estudio. Con esto se pretende tener una argumentación mucho más sólida que aquella que justifica la asignación de presupuestos más elevados para el sector, con fundamento en juicios de valor que no presentan evidencia estadística y que, por demás, caen en la tentación de los lugares comunes. Esto no implica que dichos juicios sean falsos o que se pretenda deslegitimarlos, por el contrario, lo que se busca es otorgar elementos técnicos que los respalden y sustenten.

En cuanto al contenido del presente artículo, se incluye además de esta introducción, la justificación y los objetivos del estudio para posteriormente presentar la metodología aplicada. La misma que permitió encontrar los resultados, que se presentan justo antes de finalizar con las conclusiones más relevantes.

${ }^{2}$ Este enfoque empírico tiene su trabajo pionero en Rosenbaum y Rubin (1983).

${ }^{3}$ En suma, se privilegiaron aquellos municipios que no contaban con una biblioteca registrada en la Red de Bibliotecas Públicas de la Biblioteca Nacional de Colombia. Adicionalmente, la administración local debía proporcionar un espacio adecuado para el funcionamiento de la biblioteca y garantizar su sostenibilidad y funcionamiento. A cambio, la Nación provee una dotación de al menos tres mil volúmenes y material audiovisual complementario. 


\section{JUSTIFICACIÓN Y OBJETIVOS}

Todo proyecto social busca generar un impacto positivo en el bienestar de la población que justifique la inversión de una serie de recursos. Estos proyectos buscan, por lo tanto, generar un cambio en las condiciones de vida o en los hábitos de un grupo de individuos, mediante el aumento en la oferta de ciertos bienes o servicios. Adicionalmente, como señala Mokate (1993), el éxito de un proyecto social no depende tan sólo del aumento en el stock de bienes y servicios sino, además, de la utilización efectiva que realicen los beneficiarios. Por lo tanto, en la elaboración de estos programas, proyectos o políticas, se deben considerar las características socioeconómicas de las personas beneficiarias y se requiere construir indicadores de impacto que permitan evaluar el efecto alcanzado por ellos. Sólo así, se podrá garantizar una utilización óptima de los recursos.

La evaluación aquí planteada permite identificar la efectividad de un programa social mediante la comparación del valor que toma una variable objetivo bajo dos escenarios diferentes. Básicamente, el impacto corresponde a la diferencia entre el valor que toma la variable objetivo en un grupo de tratamiento - compuesto por personas beneficiarias del programa-, versus el valor que toma esta misma variable en un grupo de control — compuesto por individuos no beneficiados.

Existen dos grandes dificultades en esta clase de análisis. En primer lugar aparece la selección de un grupo de control similar al de tratamiento. Para esto es necesaria la construcción del escenario sin proyecto, es decir, simular la situación en la que se encontrarían los beneficiarios de un programa si no hubieran participado en él. La segunda de las dificultades antes mencionadas consiste en aislar el efecto real del proyecto. Depurar el impacto alcanzado es necesario debido a la presencia de planes alternos o variables exógenas al programa que puedan generar cambios en la variable de interés.

Una de las principales ventajas de la metodología de evaluación de impacto no experimental PSM que se expone en el siguiente apartado, es que resuelve los dos problemas señalados. Con esto se podrá determinar cómo han cambiado los índices de lectura en los habitantes de los municipios beneficiados por el PNLB, en comparación con municipios similares que no han participado. De esta manera se conocerán las diferencias en el número de libros leídos anualmente por pobladores de unos y otros municipios. 


\section{Metodología}

El procedimiento $\mathrm{PSM}^{4}$ divide la muestra objeto de estudio en dos grupos. En uno de ellos se encuentran los individuos beneficiados por el programa, conocido en la literatura como el grupo de tratamiento. En el otro se ubican los no beneficiarios, también llamado grupo de control. Aunque se podría argumentar que la unidad de análisis no deben ser todos los individuos que habitan el municipio, sino sólo aquellos que manifestaron haber leído libros en el último año, se optó por la primera opción por considerarse una hipótesis más conservadora.

El método asume que si los individuos del grupo de tratamiento no hubieran participado en el programa, se comportarían de la misma manera en que lo hacen los del grupo de control. Para que este supuesto se cumpla, es necesario establecer un criterio de emparejamiento, que asegure la similitud entre individuos de tal forma que los resultados sobre la variable de interés sean comparables. Formalmente, este supuesto se puede expresar de la siguiente manera:

$$
E\left(Y_{0} \mid D=1, X\right)=E\left(Y_{0} \mid D=0, X\right)
$$

Donde $\mathrm{Y}_{0}$ corresponde al número de libros leídos en la situación sin PNLB.

$\mathrm{D}=1$ equivale a hacer parte del programa.

$\mathrm{D}=0$ equivale a no hacer parte del programa.

$\mathrm{X}=$ características que explican la probabilidad de participar en el programa

La similitud se determina a través de modelos de elección binaria (logit o probit), los cuales arrojan la probabilidad que tiene cada individuo de participar en el programa ${ }^{5}\{\mathrm{P}(\mathrm{D}$ $=1)\}$. Esta probabilidad se calcula para todos los sujetos que conforman ambos grupos, dado un conjunto $\mathrm{X}$ de variables condicionales entre las que se incluyen las características del individuo, del hogar y del municipio. El método utiliza tan sólo los individuos del grupo de tratamiento que cuentan con una persona o grupo de personas similares en el grupo de control. Esta condición se expresa en términos formales en la ecuación (2).

$$
0<P(D=1 \mid X)<1
$$

${ }^{4}$ Para profundizar en aspectos metodológicos, véase Todd (2002) y Heckman, et. al. (1997).

5 La probabilidad de participar no puede caer por fuera del intervalo $(0,1)$, donde 1 es la seguridad de participar y 0 de no participar. 
Una vez se determina cuáles individuos pueden compararse dado que tienen la misma probabilidad de participar, existen diferentes métodos de emparejamiento que permiten conocer el impacto del programa sobre la variable objetivo, en este caso, del PNLB sobre el número de libros que se leen anualmente. La ecuación (3) ilustra el modo en que el método de emparejamiento denominado «n vecinos más cercanos» según probabilidad, construye el valor de la variable objetivo de los no beneficiarios a través de un promedio.

$$
Y_{C}=\frac{\sum_{C_{i=n}}^{n} Y_{C_{i}}}{n}
$$

$Y_{C}$ corresponde al resultado promedio para los $n$ vecinos más cercanos. Para determinar el número de vecinos ( $n$ ) más apropiado, Núñez (2005) recomienda graficar el impacto encontrado con cada uno de ellos y seleccionar aquel en el cual el efecto se estabiliza. Es decir, en el que al incluir un vecino más, el promedio de la variable no cambia significativamente.

Como lo señala Cuesta (2005), el método de Kernel es similar al de $n$ vecinos más cercanos, excepto porque pondera las observaciones del grupo de control. Dicha ponderación se obtiene a partir de una función Kernel que requiere un ancho de banda. La ventaja de este método es que le da mayor peso a las observaciones más cercanas, es decir, a los individuos del grupo de control que tienen una mayor similitud con los del grupo de beneficiarios. El ancho de banda implica que se comparan sólo aquellas observaciones que están dentro de un rango determinado por una diferencia de probabilidad establecida previamente.

La ecuación (4) muestra la manera en que el impacto (I) del programa se determina a través del promedio de las diferencias entre la variable resultado para el grupo de beneficiarios $\left(Y_{B}\right)$ y la variable objetivo para el grupo de control $\left(Y_{C}\right)$. $\mathrm{H}$ es el número de individuos beneficiarios para los cuales existe un individuo o grupo de individuos similares en el grupo de control.

$$
I=\sum_{B=1}^{H_{t}}\left(Y_{B}-Y_{C}\right) / H
$$

Para estimar los intervalos de confianza que permiten conocer si el impacto sobre el número de libros leídos es estadísticamente significativo, se utilizó la técnica de bootstrapping que estima los errores estándar mediante la generación de muestras aleatorias a partir de las observaciones iniciales. 


\section{Resultados}

\subsection{La base de datos}

La base de datos se construyó a partir de la Encuesta Continua de Hogares $(\mathrm{ECH})$ del último trimestre del año 2005. Se eliminaron las encuestas realizadas en las 13 principales ciudades del país debido a que el PNLB se enfocó principalmente en municipios pequeños. Dentro de las restantes, la muestra utilizada corresponde únicamente a las encuestas de las personas que respondieron a la pregunta ¿En los últimos doce meses, leyó algún libro?. Para obtener el impacto en el número de libros leídos, se le asignó un cero a aquellas personas que respondieron no haber leído ninguno en el último año, para los demás, se eliminaron algunos valores atípicos (outliers) que señalaban un número inconsistente y demasiado alto de libros leídos. Después de realizar estos ajustes a la base de datos, en definitiva se cuenta con 20.680 observaciones.

\subsection{El modelo de participación}

Las variables del modelo de participación (probit) que se muestran en el cuadro 1, se basan en la escasa bibliografía relativa a los determinantes de la lectura. Entre los artículos revisados se puede mencionar a Ramírez y Guerra (2005), quienes desarrollan un modelo tobit estándar censurado en el que la variable dependiente es el índice de lectura. Las variables explicativas en el modelo construido por los autores son la edad, edad al cuadrado, sexo, nivel educativo, ingresos, número de libros en el hogar, visitas a bibliotecas, situación laboral y área geográfica. Todas ellas resultan ser estadísticamente significativas, es decir, el número de libros leídos está relacionado o puede estar explicado de acuerdo con estas características.

López (2008) por otra parte, basado en las teorías del «capital cultural» ${ }^{6}$, construye un modelo tobit de datos censurados, que toma como variable dependiente el número de libros leídos y como variables independientes la edad, el nivel educativo, las cantidades de libros en el hogar, el acceso a bibliotecas, el género y el ingreso. Este autor

${ }^{6}$ Para profundizar en los planteamientos de las teorías de capital cultural y learning by consuming ver Stigler, G, J. y G.S. Becker (1977) y Lévy-Garboua, L. and C. Montmarquette (1996). 
encuentra que la asistencia a bibliotecas y el nivel de escolaridad de las personas están directamente relacionados con el número de libros leídos.

García, Prieto y Fernández (1999) indagan sobre la importancia del entorno educativo en los hábitos de lectura. Para esto siguen a Lévy-Garboua y Montmarquette (1996) al considerar que el consumo de los bienes culturales es un proceso dinámico en el que las experiencias iniciales determinan los niveles de consumo futuro. De esta manera, encuentran una relación positiva entre el nivel educativo de los individuos y la formación de hábitos de lectura. Al mismo tiempo señalan el entorno familiar como otra de las variables relevantes.

Entre las variables relacionadas con los municipios, es de esperar que a mayor capacidad financiera se presente una mayor probabilidad de participar en el programa debido a que uno de los criterios para formar parte de este, consistía en la auto-postulación del municipio y en la voluntad política de los alcaldes para cumplir con los compromisos adquiridos. A partir de un análisis de desempeño fiscal adelantado por el Departamento Nacional de Planeación (DNP) para el periodo 2000-2003 se seleccionaron 6 indicadores fiscales que aparecen en la tercera columna del cuadro 1 como variables explicativas de la probabilidad de participación de los municipios en el programa.

Cuadro 1. Variables explicativas de la probabilidad de participar en el PNLB

\begin{tabular}{|c|c|c|}
\hline Individuo & Hogar & Municipio \\
\hline Edad & Número de libros en el hogar & $\begin{array}{c}\text { Índice de Gini } \\
\text { Medida concentración del ingreso }\end{array}$ \\
\hline Edad $^{2}$ & & Tasa de ruralidad \\
\hline Estado civil & & $\begin{array}{c}\text { Autofinanciación de los gastos de } \\
\text { funcionamiento }\end{array}$ \\
\hline Nivel educativo & & Magnitud de la deuda \\
\hline Condición laboral & & Dependencia de transferencias \\
\hline & & Generación de recursos propios \\
\hline & & Magnitud de la inversión \\
\hline & & Capacidad de ahorro \\
\hline
\end{tabular}

Fuente: Elaboración propia. 


\subsection{Caracterización fiscal de los municipios beneficiados}

La sostenibilidad de las inversiones es una condición necesaria en cualquier proyecto social. Las obligaciones de los municipios están relacionadas en buena parte con la disponibilidad de recursos de libre destinación. En esta dirección y con el objeto de conocer la situación financiera de los municipios, el Departamento Nacional de Planeación (DNP) construyó un índice de desempeño fiscal a partir de la agregación de un conjunto de indicadores ${ }^{7}$; éste varía entre 0 y 100, donde la calificación más alta significa excelente condición fiscal.

Un buen desempeño fiscal representa mayores posibilidades de inversión, ahorro y de cumplimiento de responsabilidades. El indicador promedio de los municipios beneficiados es de 65.31, el cual se encuentra por encima del promedio nacional de 54.7. En el Cuadro 2 se presenta el resultado promedio de los demás indicadores construidos por DNP, los que enseñan en términos generales la buena salud de las finanzas públicas de estas poblaciones.

Cuadro 2. Indicadores de desempeño fiscal promedio de los municipios beneficiados

\begin{tabular}{|l|c|}
\hline \multicolumn{1}{|c|}{ Indicadores de desempeño fiscal } & Promedio \\
\hline Autofinanciamiento & 83,75 \\
\hline Magnitud deuda & 8,74 \\
\hline Dependencia transferencias & 83,75 \\
\hline Recursos propios & 18,26 \\
\hline Inversión & 92,38 \\
\hline Ahorro & 24,88 \\
\hline Desempeño fiscal & 65,31 \\
\hline
\end{tabular}

Fuente: DNP

7 Estos indicadores corresponden a: 1) autofinanciamiento, que equivale a la proporción dentro de los recursos de libre destinación del pago de nomina y los gastos generales de operación de la administración, 2) el indicador de magnitud de la deuda, que es el porcentaje de los recursos de los municipios que respaldan la deuda, 3) el indicador dependencia de las transferencias, que exhibe la importancia que tienen las transferencias en el total de las fuentes de financiación, 4) el indicador de generación de recursos propios, señala la relación entre los ingresos tributarios y el total de los recursos, 5) el indicador magnitud de la inversión, indica el grado de inversión respecto al gasto total y finalmente 6) el indicador capacidad de ahorro, muestra el porcentaje del ahorro corriente en los ingresos corrientes de los municipios. 
A partir del indicador de desempeño fiscal, DNP elaboró 4 tipologías fiscales. De los 58 municipios beneficiados que hacen parte de la muestra, el 36\% están clasificados en la primera, el $31 \%$ en la segunda, el $28 \%$ en la tercera y el $5 \%$ restante en la cuarta. Como se observa, la gran mayoría de estos municipios hacen parte de las dos primeras. Poblaciones clasificadas en la tipología número 1 exhiben solvencia financiera, posibilidades de generar recursos propios e importantes niveles de inversión y ahorro. Las que pertenecen a la segunda presentan un equilibrio en su balance fiscal y un desempeño viable en términos fiscales. Finalmente, las últimas dos se caracterizan por una alta dependencia de las transferencias, baja capacidad de ahorro y menores posibilidades de inversión.

Gráfico 1. Tipología fiscal de los municipios beneficiados

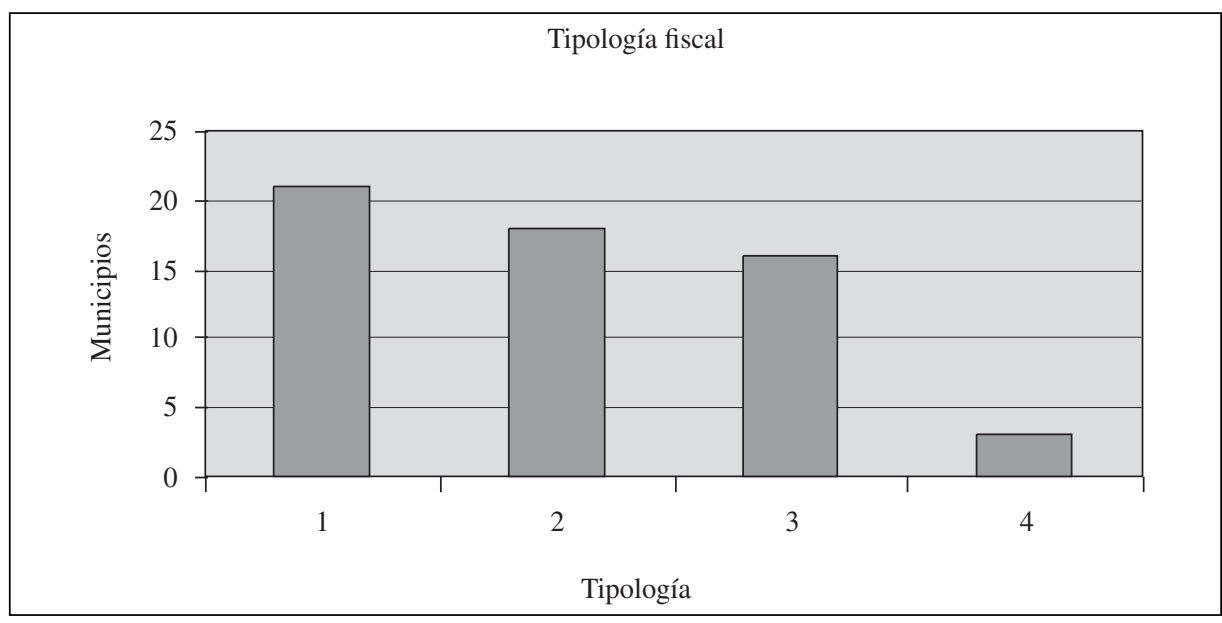

Fuente: DNP.

\subsection{Estadísticas descriptivas}

$\mathrm{Al}$ analizar algunas estadísticas descriptivas relacionadas con variables socio-económicas de los grupos de control y tratamiento se puede apreciar que los dos grupos son similares. Para mencionar unas de ellas, el promedio de edad en los municipios tratados es de 31.5 años, mientras que en municipios no beneficiarios llega a los 32.9 años, sin embargo para ambos casos, la desviación estándar no es menor a 16 años lo que indica que la población se encuentra bastante dispersa entre los diferentes rangos de edad. 
El gráfico 2 presenta el nivel educativo en los beneficiarios y los no beneficiarios, en él se observa un patrón semejante para esta variable. La mayoría de las personas cuenta con educación media y es prácticamente nula aquella porción que corresponde a personas con una escolaridad menor a la básica primaria.

Gráfico 2. Nivel educativo en los grupos de control y tratamiento
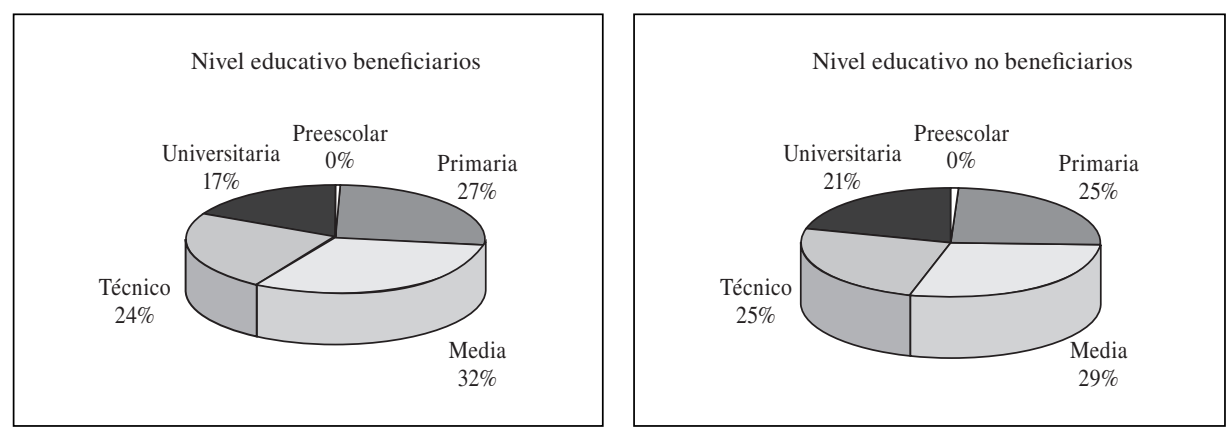

Fuente: Elaboración propia.

De igual manera, las dos poblaciones se componen en su mayoría por mujeres, siendo éstas aproximadamente el 55\% frente a un $45 \%$ de los hombres. Como se observa en el gráfico 3 el estado civil más frecuente corresponde a solteros seguido por los casados. Finalmente las principales actividades realizadas la semana anterior a la aplicación de la encuesta fueron trabajo y estudio.

\section{Gráfico 3}
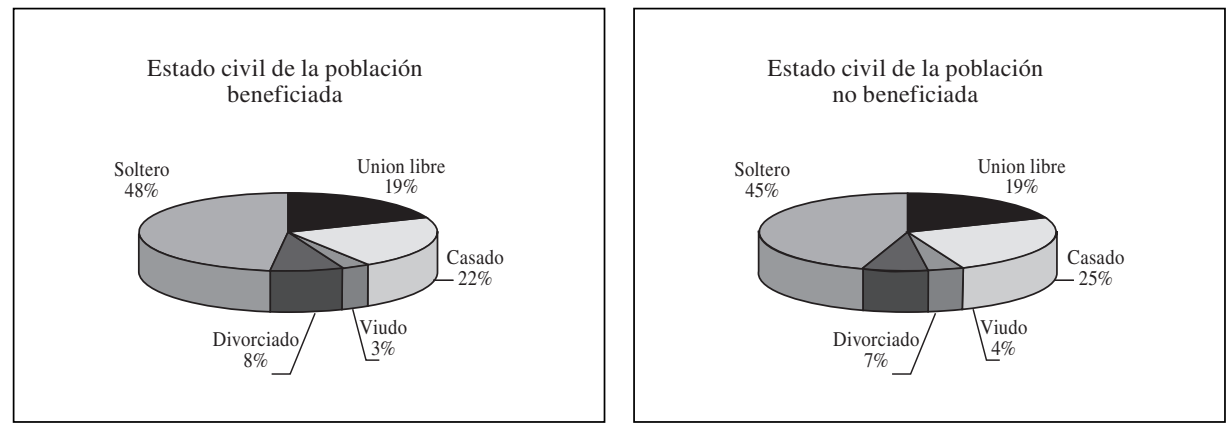

Fuente: Elaboración propia. 


\section{Gráfico 4}
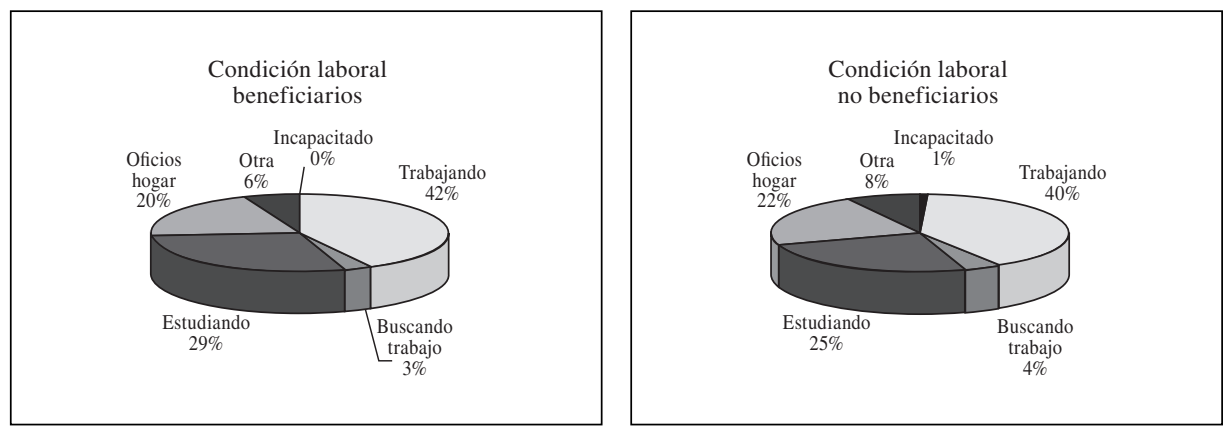

Fuente: Elaboración propia.

\section{ESTIMACIONES}

\subsection{Estimación}

El primer ejercicio que debe realizarse para determinar el impacto del PNLB sobre los hábitos de lectura es la construcción de un modelo de elección (logit o probit) como el expresado en (5). En este modelo, a partir de las variables del Cuadro 1 (del individuo, del hogar y del municipio), se determina la probabilidad de que un individuo participe en el PNLB.

$$
\begin{gathered}
P(D=1)=\beta_{0}+\beta_{1} E d a d+\beta_{2} E d a d^{2}+\beta_{3} S e x o+\beta_{4} E s t \_C i v i l+\beta_{5} N i v \_E d u c+ \\
+\beta_{6} \text { Cond_Laboral }+\beta_{7} N_{-} \text {Libros }+\beta_{8} G i n i+\beta_{9} T \_r u r a l+\beta_{10} A u t o \_f i n+ \\
+\beta_{11} \text { Mag_deuda }+\beta_{12} \text { Dep_transf }+\beta_{13} \text { Rec_prop }+\beta_{14} \text { Mag_inv }+ \\
+\beta_{15} \text { Cap_Ahorro }+e_{t}
\end{gathered}
$$

Los resultados del modelo probit son presentados en el Cuadro 3. Allí se muestra que los habitantes de municipios con capacidad de generación de recursos de libre disposición y con altos niveles de inversión y ahorro, tienen mayores posibilidades de ser beneficiarios del programa. Por el contrario los que viven en poblaciones de altos niveles de endeudamiento y baja concentración del ingreso presentan menores probabilidades de participación. 
Cuadro 3. Resultados

\begin{tabular}{|c|c|c|c|}
\hline Variable & Coeficiente & Variable & Coeficiente \\
\hline Sexo & $-0.0089213(0.021)$ & Unión libre & $\begin{array}{c}0.0778058 \\
(0.029)^{*}\end{array}$ \\
\hline Edad & $\begin{array}{c}0.0076412 \\
(0.003)^{* * *}\end{array}$ & Ninguno & $\begin{array}{c}0.0799439 \\
(0.148)\end{array}$ \\
\hline Edad $^{2}$ & $\begin{array}{l}-0.000081 \\
(0.000)^{* * *}\end{array}$ & Primaria & $\begin{array}{c}0.1004295 \\
(0.031)^{*}\end{array}$ \\
\hline Gini & $-2.445615(0.077)$ & Secundaria & $\begin{array}{c}0.1308678 \\
(0.029)^{*}\end{array}$ \\
\hline Tasa ruralidad & $0.2409176(0.044) *$ & Media & $\begin{array}{c}0.0879939 \\
(0.029)^{*}\end{array}$ \\
\hline Trabajando & $\begin{array}{c}-0.1004751 \\
(0.034)^{*}\end{array}$ & Autofinanciamiento & $\begin{array}{c}-0.0004605 \\
(0.000)\end{array}$ \\
\hline Buscando & $\begin{array}{c}-0.1785615 \\
(0.056)^{*}\end{array}$ & Magnitud deuda & $\begin{array}{c}-0.0145677 \\
(0.000)^{*}\end{array}$ \\
\hline Oficio & $\begin{array}{c}-0.1810907 \\
(0.039)^{*}\end{array}$ & Dependencia transferencias & $\begin{array}{c}0.0330234 \\
(0.001)^{*}\end{array}$ \\
\hline Otra & $\begin{array}{c}-0.2551125 \\
(0.044)^{*}\end{array}$ & Recursos propios & $\begin{array}{c}0.0291127 \\
(0.002)^{*}\end{array}$ \\
\hline Incapacitado & $-0.547371(0.166)^{*}$ & Inversión & $\begin{array}{c}0.0226427 \\
(0.002)^{*}\end{array}$ \\
\hline Soltero & $\begin{array}{c}0.0717914 \\
(0.033) * *\end{array}$ & Ahorro & $\begin{array}{c}0.0002309 \\
(0.000)^{*}\end{array}$ \\
\hline Separado & $0.1438253(0.040)^{*}$ & Libros en el hogar & $\begin{array}{c}0.000225 \\
(0.000)^{* * *} \\
\end{array}$ \\
\hline Viudo & $0.022965(0.059)$ & Constante & $\begin{array}{c}-2.922227 \\
(0.224)^{*}\end{array}$ \\
\hline Pseudo $\mathrm{R}^{2}$ & 0.1213 & $\mathrm{~N}$ & 20680 \\
\hline
\end{tabular}

Errores estándar entre paréntesis.

* Significativo al $1 \%$

** Significativo al 5\%

*** Significativo al $10 \%$

Fuente: Elaboración propia.

El modelo probit determina la probabilidad de participación para cada uno de los individuos, similares probabilidades se utilizan como criterio para emparejar beneficiarios con no beneficiarios. Los métodos de emparejamiento utilizados fueron n-vecinos y Kernel. 
Cuadro 4

\begin{tabular}{cccc}
\hline $\begin{array}{c}\text { Método de } \\
\text { emparejamiento }\end{array}$ & $\begin{array}{c}\text { Promedio de libros } \\
\text { leídos por parte de } \\
\text { beneficiarios }\end{array}$ & $\begin{array}{c}\text { Promedio de libros } \\
\text { leídos de los no } \\
\text { beneficiados }\end{array}$ & Diferencial \\
\hline 7 vecinos & 1.85 & 1.53 & 0.319 \\
Kernel & 1.85 & 1.71 & 0.142 \\
Kernel (0.01) & 1.85 & 1.57 & 0.282 \\
Kernel (0.02) & 1.85 & 1.60 & 0.254 \\
\hline
\end{tabular}

Fuente: Elaboración propia.

En el Cuadro 4 se observa que el promedio de libros leídos al año es de 1.85 para los habitantes de los municipios beneficiarios del Plan. Entre tanto, en poblaciones no beneficiadas el promedio varía entre 1.53 y 1.71, según el método de emparejamiento utilizado. Es importante señalar que independientemente de la metodología, el efecto del PNLB sobre el número de libros leídos es positivo, la diferencia radica en su magnitud.

El diferencial del índice de lectura o impacto promedio del programa sugiere que los habitantes de poblaciones beneficiadas leen entre 0.142 y 0.319 más libros que sus pares que viven en municipios que no han recibido dotaciones del PNLB. En otras palabras, según el método n-vecinos cercanos, un beneficiario del Plan Nacional de Lectura y Bibliotecas lee en promedio 0.319 más libros que una persona residente de un municipio control. Se realizó la estimación desde uno hasta diez vecinos y en este caso cuando $\mathrm{n}=7$ el efecto se estabiliza.

\section{Gráfico 5}

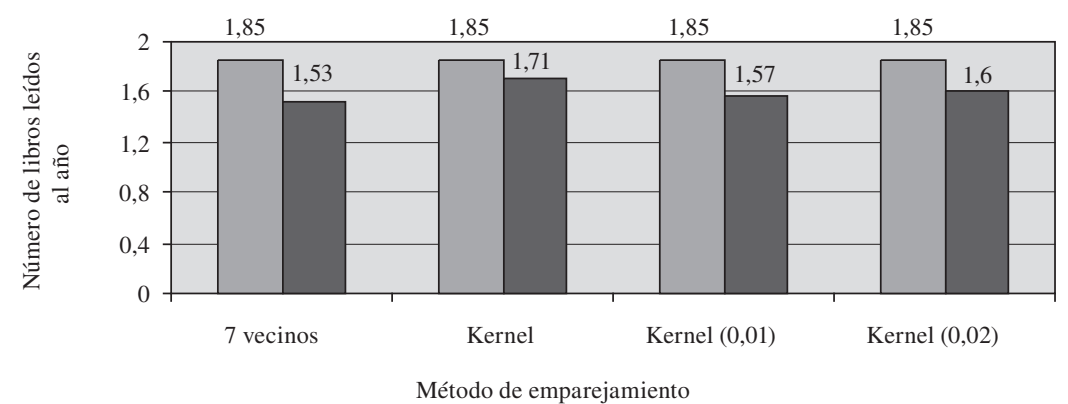

$\square$ Libros leídos al año en poblaciones beneficiadas

Libros leídos al año en poblaciones NO beneficiadas

Fuente: Elaboración propia. 
De acuerdo al Cuadro 5 ninguna de las observaciones se encuentra fuera del soporte común, es decir, para cada uno de los beneficiarios de la muestra existe un control que puede ser comparado con este individuo tratado. Esto implica que unos y otros tienen una probabilidad de participar igual o muy parecida.

Cuadro 5. Significancia estadística de los resultados

\begin{tabular}{|c|c|c|c|}
\hline Método de pareo & Diferencial & $\begin{array}{c}\text { Observaciones fuera del } \\
\text { soporte común }\end{array}$ & Significativo \\
\hline 7 vecinos & 0.319 & 0 & SI \\
\hline Kernel & 0.142 & 0 & SI \\
\hline Kernel $(0.01)$ & 0.282 & 0 & SI \\
\hline Kernel $(0.02)$ & 0.254 & 0 & SI \\
\hline
\end{tabular}

Fuente: Elaboración propia

En el Cuadro 5 se presentan los intervalos de confianza construidos mediante la técnica Bootstrapping. Los resultados son presentados para diferentes métodos estadísticos, $(\mathrm{N})$ corresponde al método clásico, en tanto que $(\mathrm{P})$ y $(\mathrm{BC})$ son métodos alternos basados en percentiles y en el método BCa. De acuerdo con estos resultados, el diferencial en el número de libros leídos estará, con un $95 \%$ de probabilidad, entre los valores presentados en las casillas de mínimo y máximo. Como se mencionó antes, en todos los métodos de emparejamiento los resultados son significativos. Esto permite concluir que efectivamente el PNLB ha tenido un impacto positivo sobre el índice de lectura en los habitantes de los municipios beneficiados.

Cuadro 6. Intervalos de confianza

\begin{tabular}{|c|c|c|c|}
\hline Metodología & Diferencial & Mínimo & Máximo \\
\hline \multirow{3}{*}{7 vecinos } & \multirow{3}{*}{0.319} & 0.1398812 & $0.4994916(\mathrm{~N})$ \\
\cline { 3 - 4 } & & 0.1519906 & $0.4778186(\mathrm{P})$ \\
\cline { 3 - 4 } & \multirow{3}{*}{ Kernel } & 0.2236714 & $0.4896223(\mathrm{BC})$ \\
\hline \multirow{2}{*}{0.142} & 0.0142609 & $0.2705836(\mathrm{~N})$ \\
\cline { 3 - 4 } & & 0.0245236 & $0.2495089(\mathrm{P})$ \\
\cline { 3 - 4 } & & 0.0383497 & $0.2982401(\mathrm{BC})$ \\
\hline
\end{tabular}


Cuadro 6 (cont.). Intervalos de confianza

\begin{tabular}{|c|c|c|c|}
\hline Metodología & Diferencial & Mínimo & Máximo \\
\hline \multirow{3}{*}{ Kernel (0.01) } & \multirow{3}{*}{0.282} & 0.1678741 & $0.3975571(\mathrm{~N})$ \\
\cline { 3 - 4 } & & 0.1541029 & $0.3665415(\mathrm{P})$ \\
\cline { 3 - 4 } & \multirow{3}{*}{0.254} & 0.1785194 & $0.3674998(\mathrm{BC})$ \\
\cline { 3 - 4 } & & 0.1374237 & $0.3712503(\mathrm{~N})$ \\
\cline { 3 - 4 } & & 0.1178199 & $0.3263299(\mathrm{P})$ \\
\cline { 3 - 4 } & & 0.1640672 & $0.3554791(\mathrm{BC})$ \\
\hline
\end{tabular}

Fuente: Elaboración propia

\section{Conclusiones y ReCOMENDACIONES}

El PNLB busca generar un cambio positivo en los hábitos de lectura de la población, se espera que resultados considerables sobre la variable de interés se aprecien en el largo plazo. Por esta razón, aunque el efecto encontrado es un avance importante debido al poco tiempo trascurrido desde la puesta en marcha del programa (tres años aproximadamente) es probable que los impactos sobre el índice de lectura, atribuibles al Plan, puedan estar siendo subestimados.

Contrastar esta hipótesis en un ejercicio posterior puede resultar bastante interesante. Encontrar cómo es el comportamiento de los impactos en diferentes momentos de tiempo, es decir, definir si los efectos del programa sobre la variable objetivo son temporales o permanentes y si el impacto año tras año se incrementa, puede generar información valiosa para la toma de decisiones por parte de las instituciones responsables del programa.

El fortalecimiento de las bibliotecas es tan sólo uno de los componentes del PNLB que pretende mejorar los hábitos de lectura de la población. Resulta importante realizar el seguimiento y una posterior evaluación de los demás componentes del programa, así como continuar con los esfuerzos de medición de los cambios en el índice de lectura.

Del artículo se puede concluir que municipios con amplia capacidad para cubrir sus gastos de funcionamiento, con altos niveles de inversión y con capacidad de ahorro tienen mayores posibilidades de decisión del gasto y de generar inversiones sostenibles como las que demanda el PNLB. Adicionalmente, a pesar de los pocos años de implementación del PNLB se evidencia un efecto positivo en uno de los componentes más relevantes del programa. Esto es, que un individuo perteneciente a un municipio 
beneficiario lee en promedio entre 0.142 y 0.319 más libros que una persona que vive en una población no beneficiada por el Plan.

Finalmente, se puede decir que el presente documento es un aporte mayúsculo para los encargados de la política cultural colombiana por cuanto aplica técnicas rigurosas en materia de evaluación de impacto. Estas técnicas suelen ser utilizadas para analizar los efectos de proyectos sociales especialmente en salud, educación y mercado laboral, pero no se conocen antecedentes de su implementación en programas de lectura. En este orden de ideas, el artículo es una novedad dentro de la literatura y hace un llamado a que se continúe con la medición de los efectos generados por otros programas culturales.

\section{REFERENCIAS BIBLIOGRÁFICAS}

CAStro, R. (2005), Metodologías y estudios de caso en la evaluación de proyectos de inversión pública en educación y salud, Universidad de los Andes, Documento interno de trabajo.

CAStro, R. y MoKATE, K. (2003), Evaluación social de proyectos de inversión, ALFAOMEGA, Bogota, D.C

Cuesta, L (2005), Impacto de los sindicatos en Colombia: ¿mayores salarios y más desigualdad?, Documento CEDE 2005-45, Universidad de los Andes, Bogotá, DC.

Dehejia, R. y Wahba, S. (1998), Propensity score matching methods for non experimental causal studies, NBER Working Paper, núm. 6829.

Departamento Nacional de Planeación (2003), Lineamientos del Plan Nacional de Lectura y Bibliotecas, Documento Conpes 3222.

- (2004). Balance del desempeño fiscal de los municipios y departamentos 2000-2003. Disponible en www.DNP.gov.co

Departamento Nacional de Planeación y Banco Mundial y Banco Interamericano de Desarrollo (2004), Programa Familias en Acción. Condiciones iniciales de los beneficiarios e impactos preliminares, Bogotá, D.C.

García, Prieto y Fernández (1999), «Los hábitos de lectura en España: Características sociales, educativas y ambientales», Revista de educación, núm. 320.

Heckman, J.; Ichimura, H. y Todd, P. (1997), «Matching as an Econometric Evaluation Estimator», Review of Economic Studies, 65 (2), 261-294.

Heckman, J.; Lalonde, R. y Smith, J. (1999), «The econometrics of active labor market programs», Handbook of labor economics, vol. 3, 1865-2097.

Lévy-Garboua, L. y Montmarquette, C. (1996). «A microeconometric study of theatre demand», Journal of Cultural Economics, 20, 25-50 
López, Omar (2008). El hábito de lectura en Colombia desde la teoría del capital de consumo, Tesis de Maestría en economía, no publicada, Universidad Nacional de Colombia, Bogotá, D.C.

Mokate, K. (1993). «La evaluación económica de los proyectos sociales», Desarrollo y Sociedad, núm. 31, marzo, CEDE, Universidad de los Andes, Bogotá, D.C.

NúÑEZ, J. (2005), Notas de clase. Mercado laboral y distribución del ingreso, Universidad de los Andes, Bogotá, D.C.

RAmírez, M. y Guerra, J. (2005), Comportamiento del lector frente a variables económicas relevantes, Memorias foro: Leer...¿Para qué? Mediciones de comportamiento del lector, CERLALC y Universidad del Rosario.

Rosenbaum, P. R. y Rubin, D. B. (1983), «The Central Role of the Propensity Score in Observational Studies for Causal Effects», Biometrika 70(1), 41-55.

Stigler, J. y Becker, G. (1977), «De gustibus non est disputandeum», American Economic Review, 67.

TodD, Petra (2002), A Practical Guide to Implementing Matching Estimators, disponible en http://athena.sas.upenn.edu/ petra

VERA-HernáNDEZ, M. (2003), «Evaluar intervenciones sanitarias sin experimentos», Gaceta Sanitaria, 17, 238-248. 